\title{
Testing Day's Conjecture that More Nitrogen Decreases Crop Yield Skewness
}

\author{
Xiaodong Du*, David A. Hennessy, and Cindy L. Yu
}

\begin{abstract}
While controversy surrounds skewness attributes of typical yield distributions, a better understanding is important for agricultural policy assessment and for crop-insurance rate setting. Day (1965) conjectured that crop yield skewness declines with an increase in nitrogen use at low levels but not at higher levels. Employing four corn yield experimental plot datasets, we investigate the conjecture by introducing $(a)$ a flexible Bayesian extension of the Just-Pope technology to incorporate skewness and $(b)$ a quantilebased measure of skewness shift. Bayesian estimation provides strong evidence in favor of negative skewness at commercial nitrogen rates and for Day's conjecture. There was weaker evidence in favor of positively skewed cotton yield and little evidence in favor of the conjecture. The results are confirmed by the quantile-based measure. We also find evidence that skewness becomes more negative upon moving from corn-after-corn to corn-after-soybean.
\end{abstract}

Key words: crop insurance, Gibbs sampler, Just and Pope technology, negative skewness, quantile regression, rotation effect.

Risks originating from the random nature of yield have significant impacts on farmers' production and marketing decisions (Goodwin and Ker 2002). A better understanding of crop yield distributions is important both for crop producers and for the crop insurance industry, where contract payout patterns are sensitive to distribution tails. There is a long-established literature on how inputs affect the mean and variance of crop yield distributions, while much less is known about how inputs affect yield skewness. A positive skew indicates that the tail on the right side is longer than the left side and the bulk of the values lie to the left of the mean.

The best-known early work on crop yield skewness is by Day (1965), whose intuition suggested positive skewness (pp. 714, 735). His rationale for the hypothesis of positive skewness reveals much about his views on the crop production process during the middle years of the 20th century (p. 714):

Xiaodong $\mathrm{Du}$ is an assistant professor in the Agricultural and Applied Economics Department, University of WisconsinMadison. David A. Hennessy is a professor in the Department of Economics and is affiliated with the Center for Agricultural and Rural Development, Iowa State University. Cindy L. Yu is an assistant professor in the Department of Statistics, Iowa State University.

*Corresponding author: 405 Taylor Hall, Department of Agricultural and Applied Economics, University of WisconsinMadison,Madison,WI53706.Tel:608-262-0699,email:xdu23@wisc. edu.
That field crop yields should conform to such a pattern seems plausible. Excellent weather condition [sic] throughout the entire growing season (particularly during the germination, flowering, heading, and harvesting season) must prevail if high yields are to be obtained. Such crop years do occur and phenomenally high yields are recorded. These pull average yields up. On the other hand, bad weather-too much or too little rain or heat-during any one of several critical periods is sufficient to reduce yields drastically, even though ideal weather is the rule during the preceding and succeeding parts of the season. Thus, common sense suggests that less than average yields are more likely than greater than average yields.

It is noteworthy that Day explored data from Mississippi, outside the main crop-growing areas of the United States, with soil and climate limitations that suggest that "ideal" conditions are not to be expected throughout the growing season. ${ }^{1}$ In addition, many have

\footnotetext{
1 Soil matters in a variety of ways, including in determining how resilient a crop is to adverse weather. Deep soils high in organic
} 
argued that improved understanding of crop nutrient needs, pest control inputs (Gardner 2002), and seed genetics ( $\mathrm{Yu}$ and Babcock 2010) have likely removed many of the factors that might constrain yield in a typical year. This is our view. We suggest further that activities intended to remove stochastic production constraints, such as increased use of nitrogen, should generally act to render skewness less positive or more negative. Crop rotation, e.g., corn-after-soybean, can also influence skewness by providing nitrogen carryover and reducing nitrogen application for the corn crop.

Day found mixed evidence on the direction of skewness. Despite some controversy in regard to the relevance of central limit theorems and concerns about methodologies used to draw inferences from aggregated data (Just and Weninger 1999; Koundouri and Kourogenis 2010), the preponderance of evidence since then has pointed to negative yield skewness for crops grown in the corn belt. See Hennessy $(2009,2010)$ for recent reviews. Much of the analysis has been on aggregated data, where input use is unknown.

We take Day's approach in scrutinizing input-controlled crop trial data and were inspired by some of his findings. For Mississippi cotton and corn experimental farm crop trial data at seven different nitrogen levels, he found a positive Pearson statistic for skewness. In the case of cotton, skewness tended to become less positive at higher nitrogen levels, while no discernible pattern emerged for corn. Oats had positive skewness at the zero nitrogen level, but negative and generally declining skewness at the higher levels. In conclusion, Day used these empirical regularities to make a tentative conjecture beyond the Mississippi Delta region (p. 739), that is, that skewness should generally decrease with increased nitrogen up to a critical level. ${ }^{2}$

For estimation of input effects on the crop yield distribution, one widely applied model is by Just and Pope $(1978,1979)$. These authors developed a stochastic production function specification that allows explicit estimation of the effect of independent variables, e.g., fertilizer, on the mean and variance of the

matter allow deep rooting and drainage away from roots. They also ensure moisture storage across the season so that early season moisture can substitute in for later water deficiencies or heat-induced water stress.

2 See point 3 in his conclusions. He does not provide any formal logical argument on why this might be so, and appears to have been surprised (p. 735). yield distribution. The 1978 paper proposes a maximum likelihood estimation procedure, while the 1979 paper proposes a three-step feasible generalized least squares approach to estimate the first and second moments of stochastic yield responses. But the model does not address yield skewness.

Antle (1983) introduces a novel momentbased approach to specifying, estimating, and testing stochastic production functions. The approach is flexible, as it imposes relatively few restrictions on the output probability distribution. Beyond mean and variance, it is also able to express third and higher moments of output as functions of inputs. The framework can be used to estimate agricultural technology as well as producers' risk-attitude parameters. For example, it is adopted by Di Falco and Chavas (2009) to investigate the effects of crop genetic diversity on farm productivity and production risk. Koundouri, Nauges, and Tzouvelekas (2006) use moments of the profit distribution to account for uncertainty in the analysis of a farmer's decisions regarding adoption of new irrigation technology. More recently, Antle (2010) has proposed and implemented a partial moment regression system approach to studying the role of inputs on skewness. For realfarm potato production data from Ecuador's Northern Highland region, he finds that fertilizer use likely decreases skewness, consistent with Day's findings but in a very different context. He finds that fungicide and labor use likely increase skewness and suggests that partial moment analysis is needed to understand subtleties in how inputs affect distribution tails.

Our empirical analysis builds on the framework of an input-conditioned beta distribution for crop yield proposed by Nelson and Preckel (1989), which has been commonly adopted in the literature. The general theme of the current study is that one should expect a relaxation of production constraints to decrease yield skewness. In addition, no further effect on skewness should be expected when the input is sufficiently large that it is unlikely to constrain. We use two approaches to investigate empirically the impact of applied nitrogen rates on skewness. In the first approach, we extend the Just-Pope model to accommodate skewness. The model is then applied to several crop yield trial experimental datasets: two datasets for corn in Iowa, one for corn in Minnesota, and one for cotton in Texas. We conduct inference within a Bayesian framework, employing the Markov chain Monte Carlo methods. 
The second empirical method involves quantile regression models to estimate skewness shifts induced by nitrogen application across different portions of a yield distribution.

We extend the well-known Just-Pope stochastic production function by introducing a skewness parameter into an originally symmetric distribution, the symmetric beta. In doing so, it is possible to retain some wellknown properties of symmetric distributions and to calculate the skewness analytically. Bayes factors, computed as the ratio of model posterior probabilities, are found to provide very strong support for our modified Just-Pope model over the regular alternative in three of the datasets, while the modified model is weakly supported in the fourth. In computation, the Bayesian estimation method applied in this study avoids the complications that often arise when optimizing a highly nonlinear maximum likelihood function. It is convenient for the proposed method to impose inequality constraints on parameters during the estimation procedure, as illustrated by Terrell (1996) and O'Donnell and Coelli (2005) in the context of imposing constraints on cost and distance functions. Quantile-based skewness measures are introduced for comparison. As we have indicated, another important advantage of the Bayesian method is that it provides a unified framework for estimation and model comparison.

After outlining the two empirical approaches, the data are explained. Then estimations are run and discussed, and some concluding comments are offered. In general, we find that corn yield skewness is positive at nitrogen levels below about $25 \mathrm{lb}$./acre, but negative at higher levels. In addition, nitrogen levels above about 75-100 lb./acre have little effect on skewness. In short, we find strong evidence in favor of Day's conjecture. For cotton, for which the fewest observations are available, we find limited evidence of positive skewness and no evidence in favor or against Day's conjecture. We also find evidence that rotation affects skewness, where corn planted after soybeans has a more negative skewness than corn planted after corn.

\section{Empirical Analysis}

We start with two facts associated with our empirical specification.

Fact 1: For $\eta$ random, if $h(\eta)$ is increasing and convex and the skewness statistics exist, then random variable $h(\eta)$ is more positively skewed than is $\eta .^{3}$

With $S(\eta)$ as the skewness statistic and $\mathbb{E}[\cdot]$ as the expectation operator, the fact can be expressed as

$$
\begin{aligned}
S(h(\eta)) & \equiv \frac{\mathbb{E}\left[(h(\eta)-\mathbb{E}[h(\eta)])^{3}\right]}{\left\{\mathbb{E}\left[(h(\eta)-\mathbb{E}[h(\eta)])^{2}\right]\right\}^{3 / 2}} \\
& \geq \frac{\mathbb{E}\left[(\eta-\mathbb{E}[\eta])^{3}\right]}{\left\{\mathbb{E}\left[(\eta-\mathbb{E}[\eta])^{2}\right]\right\}^{3 / 2}} \equiv S(\eta)
\end{aligned}
$$

In the Supplemental Materials the following is demonstrated.

Fact 2: When $h(\eta)$ is increasing and concave, then inequality (1) is reversed.

Van Zwet's (1964) observation was used by Hennessy (2010) to show a possible relationship between weather and yield distributions. Suppose that, all else equal, better weather in some index sense improves yield but the marginal effect is decreasing. Then the weather-conditioned yield distribution will be more negatively skewed than the weather distribution itself. In this study, our interest is in the role that market inputs play in determining skewness.

In the analysis to follow, we will use two distinct approaches to provide evidence on how inputs affect yield skewness. The first extends the Just-Pope specification to account for skewness and uses Bayesian methods to implement the approach. The second method invokes a quantile regression to study how the quantile gaps stretch or contract as the input changes.

\section{Bayesian Analysis of a Skewness Measure}

Let experimental plot crop yield $y$ be given by

$$
y=f(z)+g(z) \varepsilon^{h(z)}
$$

where $z$ is an input, e.g., nitrogen. Here $\varepsilon$ is random, while $f(\cdot), g(\cdot)$, and $h(\cdot)$ are functions to be parameterized and estimated. To be consistent with Just-Pope applications, let

$$
\begin{aligned}
& f(z)=\alpha_{0} z^{\alpha_{1}} ; \quad g(z)=\beta_{0} z^{\beta_{1}} ; \\
& h(z)=\gamma_{0} z^{\gamma_{1}} .
\end{aligned}
$$

\footnotetext{
3 Theorem 2.1.1, van Zwet (1964), p. 10.
} 
The moments for crop yield are:

$$
\begin{aligned}
& \mathbb{E}[y]=\alpha_{0} z^{\alpha_{1}}+\beta_{0} z^{\beta_{1}} \mathbb{E}\left[\varepsilon^{\gamma_{0} z^{\gamma_{1}}}\right] ; \\
& \mathbb{E}\left[(y-\mathbb{E}[y])^{2}\right]=\beta_{0}^{2} z^{2 \beta_{1}} \operatorname{Var}\left(\varepsilon^{\gamma_{0} z^{\gamma_{1}}}\right) ; \\
& S(y)=\frac{\mathbb{E}\left[\left(\varepsilon^{\gamma_{0} z^{\gamma_{1}}}-\mathbb{E}\left[\varepsilon^{\gamma_{0} z^{\gamma_{1}}}\right]\right)^{3}\right]}{\left\{\mathbb{E}\left[\left(\varepsilon^{\gamma_{0} z^{\gamma_{1}}}-\mathbb{E}\left[\varepsilon^{\gamma_{0} z^{\gamma_{1}}}\right]\right)^{2}\right]\right\}^{3 / 2}} .
\end{aligned}
$$

Equation (4) implies that (a) skewness is determined by $\left(\gamma_{0}, \gamma_{1}\right)$ only; $(b)$ scale (and so yield variance $)$ is determined by $\left(\beta_{0}, \beta_{1}, \gamma_{0}, \gamma_{1}\right)$ so that scale can change with the level of $z$ independent of skewness; $(c)$ location (and so yield mean $)$ is determined by $\left(\alpha_{0}, \alpha_{1}, \beta_{0}, \beta_{1}, \gamma_{0}, \gamma_{1}\right)$ so that location can change with $z$ independent of yield variance and skewness. In summary, the stochastic production function in equation (2) is flexible for mean, variance, and skewness.

We estimate the stochastic production technology using crop nitrogen trial data. Given the discrete and limited number of nitrogen levels applied in such trials, we estimate the impacts of the nitrogen input on the skewness of crop yield distribution for each nitrogen level individually. For the nitrogen application level $i$, $i \in \Omega_{I} \equiv\{1,2, \ldots, I\}$, we adapt equation (2) to give our empirical crop yield model as: ${ }^{4}$

$$
\begin{aligned}
y^{i}= & a_{0}^{i}+\mathbf{X} \boldsymbol{\beta}+b^{i} \varepsilon^{1 / c^{i}}, \\
\varepsilon & \sim D \times \operatorname{Beta}(\alpha, \alpha) ; \quad i \in \Omega_{I} .
\end{aligned}
$$

where $y^{i}=\left(y_{1}^{i}, y_{2}^{i}, \ldots, y_{k}^{i}\right)^{\prime}$ denotes the $k$ plot-level crop yield observations for the $i$ th nitrogen level. Parameter $a_{0}^{i}$ denotes the constant term in the yield equation where it is allowed to vary with nitrogen application, and it includes the effect of nitrogen on mean yield, $f\left(z^{i}\right)$. Matrix $\mathbf{X}=\left(x_{1} x_{2} \ldots x_{L}\right)$, in which $x_{l}=\left(x_{l 1}, x_{l 2}, \ldots, x_{l I}\right)^{\prime} \forall l \in \Omega_{L}$, denotes controlled variables such as location, rotation, and/or tillage effect, and technological innovation in crop production represented by

\footnotetext{
${ }^{4}$ The probability density function $(p d f)$ of the general beta distribution is

$$
\begin{aligned}
p\left(x ; \alpha_{1}, \alpha_{2}\right) & =\frac{\Gamma\left(\alpha_{1}+\alpha_{2}\right)}{\Gamma\left(\alpha_{1}\right) \Gamma\left(\alpha_{2}\right)} x^{\alpha_{1}-1}(1-x)^{\alpha_{2}-1} \\
& =\frac{1}{B\left(\alpha_{1}, \alpha_{2}\right)} x^{\alpha_{1}-1}(1-x)^{\alpha_{2}-1},
\end{aligned}
$$

where $\Gamma(\cdot)$ is the gamma function and the beta function $B\left(\alpha_{1}, \alpha_{2}\right)$ appears as a normalization constant. The above $p d f$ is defined on the interval $(0,1)$ with two positive shape parameters, $\alpha_{1}$ and $\alpha_{2}$. Instead we employ a symmetric beta distribution, $\operatorname{Beta}(\alpha, \alpha)$, which is parameterized by $\alpha\left(=\alpha_{1}=\alpha_{2}\right)$.
}

time dummy or trend. The corresponding coefficient vector is $\boldsymbol{\beta}=\left(\beta_{1}, \beta_{2}, \ldots, \beta_{L}\right)^{\prime}$. The scale effect in specification (2) is represented by $b^{i}=g\left(z^{i}\right)$.

The symmetric beta distribution for the random error $\varepsilon$ is a building block of the model. $D$ is specified as a constant across nitrogen levels and is used to scale up the support of the beta distribution from $[0,1]$ to $[0, D]$. As $b^{i}$ varies with and $D$ is constant across nitrogen application level $i$, both parameters are identifiable in the current setting, which is confirmed by the estimation results we will discuss later.

We specify the yield random variations as $\varepsilon^{1 / c^{i}}$ with skewness parameter $c^{i}$ and assume that $\varepsilon$ follows a symmetric beta distribution $\operatorname{Beta}(\alpha, \alpha)$ on the range $[0, D]$. Based on the constructive representation in equation (5), skewness is introduced into an originally symmetric distribution on $\varepsilon$ through parameter $c^{i}$. Doing so allows us to retain some well-known properties of symmetric distributions, so that Fact 1 and Fact 2 allow skewness to be ordered in a common framework. Notice that transformation $h(\varepsilon)=\varepsilon^{1 / c^{i}}$ is increasing whenever $c^{i}>$ 0 , and also that it has a uniformly signed second derivative. The symmetric specification of $\varepsilon$ in equation (5), together with Fact 1 and Fact 2 , mean that condition $0<c^{i}<1$ (i.e., $1 / c^{i}>1$ ) implies a positive yield skew at the $i$ th nitrogen level. On the other hand, condition $c^{i}>1$ (i.e., $0<1 / c^{i}<1$ ) implies a negative skew. In addition, higher $c^{i}$ is associated with decreasing skewness.

Inference here is conducted within a Bayesian framework. The Bayesian approach allows us to incorporate critical inequality constraints on parameters into the estimation procedure. Parameter estimates must satisfy $0<\left[\left(y^{i}-a_{0}^{i}-\mathbf{X} \boldsymbol{\beta}\right) / b^{i}\right]^{c^{i}}<D$ to be consistent with assumptions on the underlying distribution. Furthermore, the Bayesian estimation procedure is particularly suitable because the model specified in equation (5) is very nonlinear in the parameters. When compared with the maximum likelihood estimator, our proposed Bayesian method is easier to implement, where we use Markov chain Monte Carlo methods to draw from conditional posterior distributions of parameters.

For the parameter vector in model (5), $\Theta=\left\{\mathbf{a}_{\mathbf{0}}, \boldsymbol{\beta}, \mathbf{b}, \mathbf{c}, D, \alpha\right\}$, where $\mathbf{a}_{\mathbf{0}}=\left\{a_{0}^{i}\right\}_{i=1}^{I}, \mathbf{b}=$ $\left\{b^{i}\right\}_{i=1}^{I}, \mathbf{c}=\left\{c^{i}\right\}_{i=1}^{I}$, Bayesian inference stems from the joint posterior distribution of the model parameters conditional on the observed 
data, which can be expressed as follows:

$$
\begin{aligned}
p\left(\mathbf{a}_{\mathbf{0}}, \boldsymbol{\beta}, \mathbf{b}, \mathbf{c}, D, \alpha \mid Y, \mathbf{X}\right) & \\
\propto & {\left[\frac{1}{D^{\alpha} B(\alpha, \alpha)}\right]^{N} \prod_{i=1}^{I} \prod_{k=1}^{n_{i}} \frac{c^{i}}{b^{i}} } \\
& \times\left(\frac{y_{k}^{i}-a^{i}}{b^{i}}\right)^{c^{i} \alpha-1} \\
& \times\left[1-\frac{1}{D}\left(\frac{y_{k}^{i}-a^{i}}{b^{i}}\right)^{c^{i}}\right]^{\alpha-1} \\
& \times I_{A} \times p(\Theta) ; \\
a^{i}= & a_{0}^{i}+\mathbf{X} \boldsymbol{\beta} ; \\
I_{A}= & \begin{cases}1 \quad \text { if } \frac{1}{D}\left(\frac{y_{k}^{i}-a^{i}}{b^{i}}\right)^{c^{i}} \in(0,1), \\
0 \quad \text { otherwise. }\end{cases}
\end{aligned}
$$

where $N\left(=\sum_{i=1}^{I} n_{i}\right)$ is the total observation number and $B(\alpha, \alpha)\left(=\int_{0}^{1} t^{\alpha-1}(1-t)^{\alpha-1} d t\right)$ denotes the beta function. The first three items are the likelihood function, while the last term in the product, $p(\Theta)$, denotes the joint prior distribution of model parameters.

The Gibbs sampler, one of the Markov chain Monte Carlo methods in Bayesian implementation, is applied by repeated sampling from the conditional posterior density of each parameter. It is obvious that the posterior distribution for each parameter derived from the joint posterior distribution in equation (6) does not belong to any convenient distribution family. Thus we employ the random-walk MetropolisHasting algorithms (see the detailed introduction and application by, e.g., Gelman et al. 2007; Koop, Poirier, and Tobias 2007) for updating draws from each posterior distribution of model parameters. Technical details regarding the conditional posterior distributions and implementation of the Gibbs sampler are provided in the Supplemental Materials. After convergence, the draws of the Gibbs sampler for each parameter are used to compute the mean and standard deviation of the marginal posterior distribution.

\section{Quantile Regression Analysis of Skewness Shifts}

Quantile-based measures can be used to describe shape-shifts of a distribution, including skewness (Hao and Naiman 2007, ch. 2). Note that for a skewed distribution, the quantile function $Q^{(p)}$, which is defined as $P\left(Z \leq Q^{\left(p_{i}\right)}\right)=p_{i}$ for a random variable $Z$ and probability $p_{i} \in[0,1]$, is asymmetric around the median. Based on a random sample, the quantile-based measure, $S^{(p)}$, defined as the ratio of the spreads above and below the median (upper spread vs. lower spread $), S^{(p)}=\left[\left(Q^{(1-p)}-\right.\right.$ $\left.\left.Q^{(0.5)}\right) /\left(Q^{(0.5)}-Q^{(p)}\right)-1\right]$ for $p<0.5$ could be used to measure skewness (Hao and Naiman 2007). ${ }^{5}$ For symmetric distributions, $Q^{(1-p)}-$ $Q^{(0.5)}=Q^{(0.5)}-Q^{(p)}$ so that $S^{(p)}<0$ means that the concave relation in $p, Q^{(1-p)}-Q^{(0.5)}<$ $Q^{(0.5)}-Q^{(p)}$, applies, and this is consistent in spirit with Fact 2.

Compared with a reference case, disproportional upper and lower spread changes relative to the median indicate skewness shifts in a comparison case. Thus the sample-based skewness shift, $S S^{(p)}$, for the middle $100(1-2 p) \%$ of the population is defined as

$$
\begin{aligned}
S S^{(p)}= & {\left[\begin{array}{l}
\left(Q_{C}^{(1-p)}-Q_{C}^{(0.5)}\right) / \\
\frac{\left(Q_{R}^{(1-p)}-Q_{R}^{(0.5)}\right)}{\left(Q_{C}^{(0.5)}-Q_{C}^{(p)}\right) /}-1 \\
\left(Q_{R}^{(0.5)}-Q_{R}^{(p)}\right)
\end{array}\right] } \\
& \text { for } p<0.5
\end{aligned}
$$

(Hao and Naiman 2007). ${ }^{6}$ Here $Q_{C}^{(p)}$ and $Q_{R}^{(p)}$ are the $p$ quantiles for the comparison and reference cases, respectively.

In this study, the quantile regression model (QRM) introduced by Koenker and Bassett (1978) is employed to characterize the impact of explanatory variables, e.g., nitrogen, on the shape of the yield distribution. The model is specified as

$$
\begin{gathered}
y_{i}^{(p)}=X^{\prime} \gamma^{(p)}+\varepsilon_{i}^{(p)}=\alpha^{(p)}+\gamma_{z}^{(p)} z_{i} \\
+X_{\{-z\}}^{\prime} \gamma_{\{-z\}}^{(p)}+\varepsilon_{i}^{(p)}, \\
\operatorname{Quan}_{p}\left(y_{i} \mid X\right)=X^{\prime} \gamma^{(p)}, \quad i \in \Omega_{I} .
\end{gathered}
$$

where Quan ${ }_{p}\left(y_{i} \mid X\right)=X^{\prime} \gamma^{(p)}$ denotes the quantile of crop yield, $y_{i}$, conditional on a set of controlled variables, $\mathbf{X}=\left(z X_{\{-z\}}\right)$, in which the first variable is the nitrogen application rates, $z$, while $X_{\{-z\}}^{\prime}=\left(x_{2} \ldots x_{L}\right)$ are other controlled variables as defined in equation (5).

\footnotetext{
5 Equation 2.2, p. 14

${ }^{6}$ Equation 5.2,p. 72. We do not calculate sample-based skewness shift $S S^{(p)}$ in this study. Instead, we employ and report model-based measure $S K S^{(p)}$ at a later juncture.
} 
All controlled variables are centered at sample means.

Following the measure in equation (7), the skewness shift resulting from one more unit of additional nitrogen application can be calculated from the estimated QRM coefficients: ${ }^{7}$

$$
\begin{aligned}
& \operatorname{SKS}^{(p)} \\
& \quad=1000\left[\begin{array}{l}
\left(\hat{\gamma}_{z}^{(1-p)}+\hat{\alpha}^{(1-p)}-\hat{\gamma}^{(0.5)}\right. \\
\frac{\left.-\hat{\alpha}^{(0.5)}\right) /\left(\hat{\alpha}^{(1-p)}-\hat{\alpha}^{(0.5)}\right)}{\left(\hat{\gamma}_{z}^{(0.5)}+\hat{\alpha}^{(0.5)}-\hat{\gamma}_{z}^{(p)}\right.}-1 \\
\left.-\hat{\alpha}^{(p)}\right) /\left(\hat{\alpha}^{(0.5)}-\hat{\alpha}^{(p)}\right)
\end{array}\right]
\end{aligned}
$$$$
\text { for } p<0.5
$$

where the estimated constant values of $\hat{\alpha}$ provide the fitted quantile functions at the means of independent variables and are defined as the reference cases. Standard errors of the estimated skewness shifts can be obtained by the bootstrap method (Greene 2003, p. 924). For a given portion, $100(1-2 p) \%$, of the yield population, $S K S^{(p)}<0$ indicates a reduction of right-skewness induced by an increase of nitrogen application, after controlling for other covariates. Conversely, $S K S^{(p)}>0$ indicates an exacerbation of right-skewness, i.e., the crop yield is more positively/right skewed.

\section{Data}

In this study, four crop yield datasets A, B, C, and D are employed. We do not consider Day's data because, apart from the different methodologies we bring to bear, that would amount to in-sample validation of the conjecture. One common feature of the datasets is that all have been collected from controlled experiments, although the experiments are conducted under different control conditions such as tillage practices and rotation sequences.

Dataset A: Corn production data are obtained from controlled experiments conducted at Iowa State University's Research and Demonstration Farm located in Floyd County, Iowa, from 1979 to 2003 (Mallarino, Ortiz-Torres, and Pecinovsky 2004). The corn yield data are collected under four rotations, $<\mathrm{C}>,<\mathrm{CS}>,<\mathrm{CCS}>$, and $<\mathrm{CCCS}>$, where $<\mathrm{CCCS}>$ is to be read as the corn-corncorn-soybeans rotation. Four levels of nitrogen

\footnotetext{
${ }^{7}$ Equation 5.2, p. 72 in Hao and Naiman (2007). We have introduced the factor 1000 because quantile bound $p \in[0,1]$ leads to very small numbers.
}

treatments-0, 80, 160, and $240 \mathrm{lb}$./acre-are applied for corn. Each combination of rotation and nitrogen level is replicated three times a year. Using the same dataset, Hennessy (2006) investigates the rotation effect on crop yield and on nitrogen input choices. Rotation effect is found to persist for one year. Taking the one-year memory into account, dataset A contains 525 observations for each nitrogen level. The corresponding specification for equation (5) after including the controlled variables is $y^{i}=a_{0}^{i}+\sum_{j=1}^{2} x_{j} \beta_{j}+b^{i} \varepsilon^{1 / c^{i}} ; i=1,2,3,4$; where $\boldsymbol{\beta}=\left[\beta_{1}, \beta_{2}\right]^{\prime}$ represents the average effects of crop rotation $<\mathrm{CC}>$ and linear time trend on corn yields. Here and when modeling the datasets to follow, we remind the reader that $\varepsilon \sim D \times \operatorname{Beta}(\alpha, \alpha)$, as laid out in equation (5).

Dataset $B$ : Corn yields are generated by controlled experiments on fifteen geographically dispersed Iowa farms under $<\mathrm{CC}>$ and $<\mathrm{CS}>$ rotations from 1985 to 1990 under ten levels of nitrogen fertilizer: $0,25,50,75,100,120$, 150, 200, 250, and $300 \mathrm{lb}$./acre. In the context of yield and revenue insurance, Babcock and Hennessy (1996) employ part of this dataset to estimate conditional distributions of crop yields. There are three replications of each nitrogen level. Total observations for each level of nitrogen vary from 193 to 203. The control variables for equation (5) include five dummies, $\beta_{1}, \ldots, \beta_{5}$, for the years $1985,1986,1987$, 1988 , and 1989; one dummy $\beta_{6}$ for the CC rotation; and fourteen dummy variables for farm locations $\left(\beta_{7}, \ldots, \beta_{20}\right)$. The corresponding specification is $y^{i}=a_{0}^{i}+\sum_{j=1}^{20} x_{j} \beta_{j}+b^{i} \varepsilon^{1 / c^{i}}$; $i=1,2, \ldots, 10$.

Dataset C: Corn yield data are collected from replicated plot experiments in Morris County, Minnesota, from 1985 to 1989 with five nitrogen fertilizer rates of $0,40,80,120$, and $160 \mathrm{lb}$./acre. ${ }^{8}$ For each nitrogen rate, there are 167 or 168 observations. Besides the time indicators for 1985-1988 $\left(\beta_{1}, \ldots, \beta_{4}\right)$, dummies for various tillage practices, fall plow $\left(\mathrm{FP} ; \beta_{5}\right)$, fall chisel $\left(\mathrm{FC} ; \beta_{6}\right)$, and ridge till $\left(\mathrm{RD} ; \beta_{7}\right)$ are also included as controlled variables in the equation (5) regression, which is specified as

$$
\begin{gathered}
y^{i}=a_{0}^{i}+\sum_{j=1}^{7} x_{j} \beta_{j}+b^{i} \varepsilon^{1 / c^{i}} ; \\
i=1,2, \ldots, 5 .
\end{gathered}
$$

\footnotetext{
${ }^{8}$ See Mitchell (2004) for more details on the dataset. We thank Paul Mitchell for providing us with datasets C and D.
} 
Dataset D: We choose to consider cotton yield data because the crop has received some attention in the skewness literature. Ramirez, Mishra, and Field (2003) studied dryland cotton in the West Texas Plains counties of Childress, Cochran, Crosby, Hale, and Wichita. Like Day, these authors identify positive skewness. Similar to Day's intuition, they ascribe it to the positive skewness of the limiting factor, rainfall. Our cotton yield data are obtained from field experiments in Calhoun, San Patricio, and Wharton counties in the Texas Coastal Bend cotton growing region for 1998-2002. ${ }^{9}$ Nitrogen fertilizer are applied at four levels: 0, 50, 100 , and $150 \mathrm{lb}$./acre. The controlled variables in equation (5) are two location dummies for the first two sampling areas, $\beta_{1}$ and $\beta_{2}$, and time dummies for the years 1998-2001 $\left(\beta_{3}, \ldots, \beta_{6}\right)$. There are thirty-nine yield observations for each nitrogen rate. The regression model is specified as:

$$
\begin{gathered}
y^{i}=a_{0}^{i}+\sum_{j=1}^{6} x_{j} \beta_{j}+b^{i} \varepsilon^{1 / c^{i}} ; \\
i=1,2, \ldots, 4 .
\end{gathered}
$$

\section{Parameter Estimates and Discussion of Results}

\section{Bayesian Analysis of a Skewness Measure}

The Gibbs sampler is fully documented in the Supplemental Materials and is coded in Matlab. We run the posterior simulator for 10,000 simulations and discard the first 5,000 simulations as burn-in. Estimation results on generated data experiments revealed that the Bayesian algorithm is able to recover parameters of the data generation process well. The priors of the model parameters are set to be reasonably non-informative as follows: $a_{0}^{i} \sim$ $N(10,10) ; b^{i} \sim N(100,20) ; c^{i} \sim N(1,1) ; D \sim$ $N(0,10) ; \alpha \sim N(0,10)$; and $\beta_{i} \sim N(0,10)$. For each of datasets A, B, C, and D, the Supplemental Materials present coefficient posterior means, standard deviations, and model parameter probabilities of being positive.

\footnotetext{
${ }^{9}$ The original data do not provide irrigation information. Comparing the yields in the data with the county yields and typical dryland vs. irrigated yields of the National Agricultural Statistics Service, it is clear that the yields are dryland cotton. We thank Paul Mitchell for pointing this out. See Seo, Mitchell, and Leatham (2005) for more details on the dataset.
}

Following equation (4), the skewness of conditional corn yield distribution for each nitrogen application level is calculated as

$$
\begin{aligned}
& S\left(y^{i}\right)= \frac{\mathbb{E}\left[\left(\varepsilon^{d^{i}}-\mathbb{E}\left[\varepsilon^{d^{i}}\right]\right)^{3}\right]}{\left\{\mathbb{E}\left[\left(\varepsilon^{d^{i}}-\mathbb{E}\left[\varepsilon^{d^{i}}\right]\right)^{2}\right]\right\}^{3 / 2}} \\
& \frac{B\left(\alpha+3 d^{i}, \alpha\right)}{B(\alpha, \alpha)}-\frac{3 B\left(\alpha+2 d^{i}, \alpha\right) B\left(\alpha+d^{i}, \alpha\right)}{[B(\alpha, \alpha)]^{2}} \\
&=\frac{+2\left(\frac{B\left(\alpha+d^{i}, \alpha\right)}{B(\alpha, \alpha)}\right)^{3}}{\frac{B\left(\alpha+2 d^{i}, \alpha\right)}{B(\alpha, \alpha)}-\left(\frac{B\left(\alpha+d^{i}, \alpha\right)}{B(\alpha, \alpha)}\right)^{2}},
\end{aligned}
$$

where $d^{i}=1 / c^{i}$. The mean and standard errors of skewness for each nitrogen level are calculated using the draws from posterior distributions of individual parameters and are presented in table 1.

It can be seen from the results in table 1 that for datasets $\mathrm{A}, \mathrm{B}$, and $\mathrm{C}$ the estimated skewness measures are generally consistent with the conjecture and prior expectation. An increase in nitrogen inputs makes average corn production more negatively skewed. While positive skewness is shown at zero or low levels of nitrogen rates, skewness becomes negative at higher nitrogen levels. Skewness ceases to decrease further in response to an increase in nitrogen at levels more than approximately $100 \mathrm{lb}$./acre.

Specifically, in dataset A, we see strong evidence of negative skewness associated with nonzero nitrogen rates. The posterior standard deviations for the skewness parameters are quite small relative to their means. An increase in nitrogen is associated with an increase in the absolute values of skewness. In datasets B and $\mathrm{C}$, we see similar patterns for skewness shifts, but the inference from these data are not as strong given that the posterior standard deviations are of nearly equal magnitude to the posterior means.

For cotton yield in dataset D, positive skewness is found for all nitrogen rates. The finding is consistent with findings in the literature (e.g., Day 1965; Ramirez, Misra, and Field 2003). As pointed out in some other studies, the positive skewness of cotton yield could be related to rainfall and drought conditions in the areas under scrutiny. In other words, nitrogen is generally not the limiting factor.

\section{Comparison with the Regular Just-Pope Model}

Bayes factors are used widely for model comparison in Bayesian framework. They are 
Table 1. Bayesian Posterior Mean and Standard Deviation (in parentheses) for Derived Mean, Variance, and Skewness

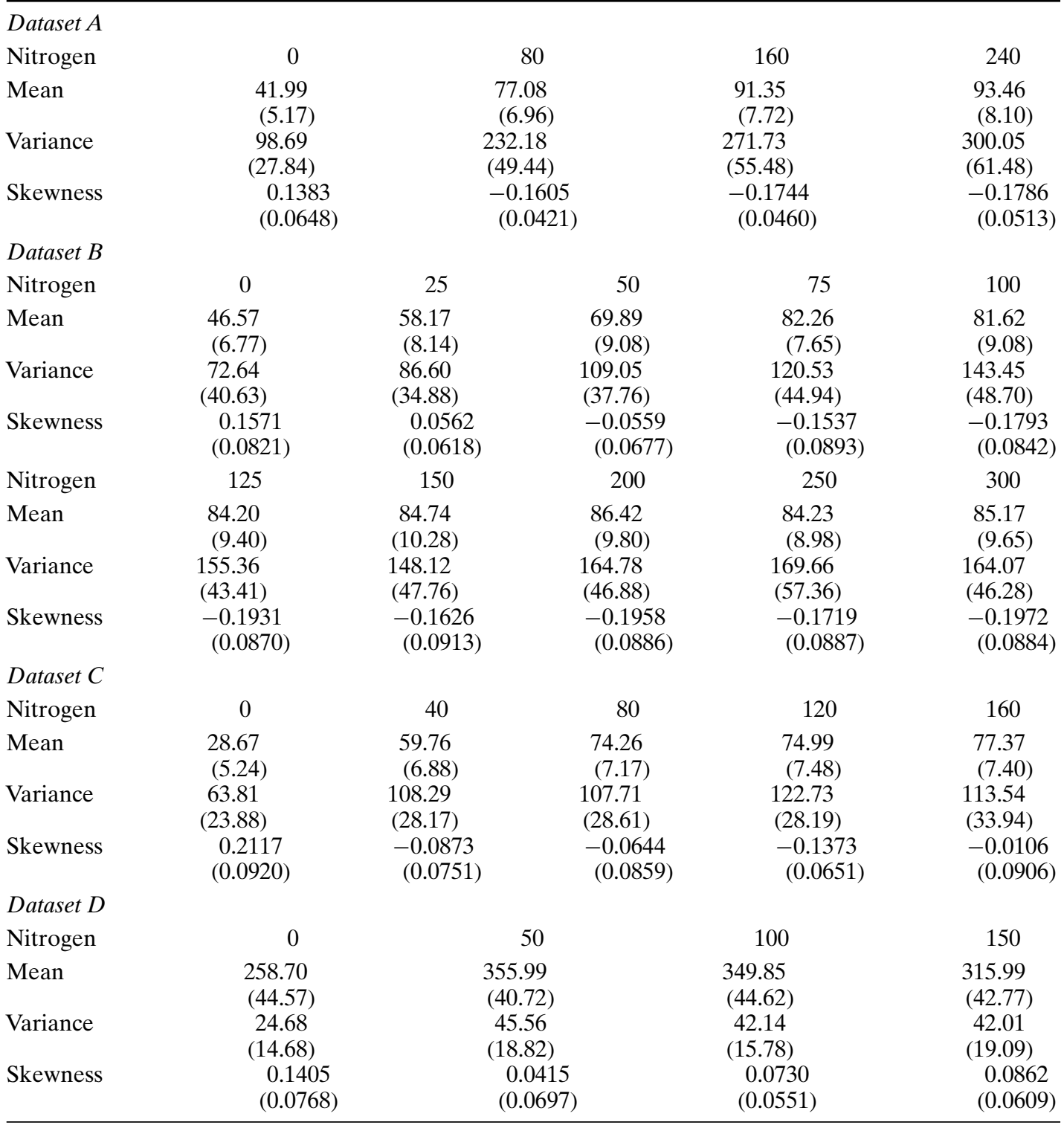

the Bayesian alternative to likelihood ratio tests. Bayes factors are flexible, allowing easy comparison of nonnested models and simultaneous comparison of multiple hypotheses. Another advantage of the Bayes factor is that it includes an implicit and automatic penalty for model complexity without active calculation of the number of parameters (Kass and Raftery 1995). Bayes factors summarize evidences provided by data and thus are critical for model comparison.

By Bayes' theorem, the posterior probability of a model $M_{k}$ given data $y$ can be obtained from the marginal likelihood $p\left(y \mid M_{k}\right)$ and the prior probability $p\left(M_{k}\right)$, as $p\left(M_{k} \mid y\right)=p\left(y \mid M_{k}\right) p\left(M_{k}\right) / p(y)$. A Bayes factor is defined as the ratio of two posterior probabilities of models $M_{1}$ and $M_{2}$, i.e., $\frac{p\left(M_{1} \mid y\right)}{p\left(M_{2} \mid y\right)}=$ $\frac{p\left(y \mid M_{1}\right)}{p\left(y \mid M_{2}\right)} \times \frac{p\left(M_{1}\right)}{p\left(M_{2}\right)}$, where $p\left(M_{k}\right)$ (for $\left.k \in\{1,2\}\right)$ is the prior for model $k$. To ensure a fair comparison, equal priors are always assumed for the models, i.e., $p\left(M_{k}\right)=0.5$. Under this case, the Bayes factor becomes a ratio of two marginal likelihoods $p\left(M_{1} \mid y\right) / p\left(M_{2} \mid y\right)$, where the marginal likelihood under model $k$ is 
Table 2. Bayes Factors of the Modified Just-Pope Model $\left(M_{1}\right)$ relative to the Regular Just-Pope Model $\left(M_{2}\right)$

\begin{tabular}{lclcc}
\hline $2 \log _{e}\left(B_{12}\right)$ & Dataset A & Dataset B & Dataset C & Dataset D \\
\hline & 235.10 & 284.68 & 116.34 & 4.84 \\
\hline $2 \log _{e}\left(B_{12}\right)$ & & Evidence favoring model 1 & \\
0 to 2 & & Not worth more than a bare mention & \\
2 to 6 & & Positive & \\
6 to 10 & & Strong & \\
$>10$ & & Very strong & \\
\hline
\end{tabular}

Note: Interpretation (Kass and Raftery 1995).

defined as $p\left(y \mid M_{k}\right)=\int p\left(y \mid \theta, M_{k}\right) p\left(\theta \mid M_{k}\right) d \theta$, and could be interpreted as the "probability of seeing the data that actually were observed" (Kass and Raftery 1995).

To compare with our extended Just-Pope model, we fit the regular Just-Pope type of model to the same datasets where the model is defined as

(11) $y^{i}=\bar{a}_{0}^{i}+\mathbf{X} \boldsymbol{\beta}+\bar{b}^{i} \zeta, \zeta \sim \operatorname{Beta}(\bar{\alpha}, \bar{\alpha}) ; i \in \Omega_{I}$.

The model specified in equation (11) is the same as equation (5) except for the skewness measure $1 / c^{i}$ and the parameter $D$, where the latter is now captured by the new scale factor $\bar{b}^{i}$. The joint posterior distribution and the Gibbs sampler can be easily derived following the approach described in equation (6) and in the Supplemental Materials. We denote the modified Just-Pope model in equation (5) as model 1 and the regular Just-Pope model in equation (11) as model 2.

For the purpose of model comparison, Bayes factors are computed using the method described by Kass and Raftery (1995). Specifically, for each model ( $k=1$ or 2$)$, we use the posterior draws (after burn-in period) produced from the Gibbs sampler of model $k$ to obtain an estimate of the marginal likelihood $\widehat{p r}\left(\mathbf{y} \mid M_{k}\right)$ for the crop yield produced under reference conditions (i.e., setting all covariates effects $\beta$ to zero), ${ }^{10}$

$$
\widehat{\operatorname{pr}}\left(\mathbf{y} \mid M_{k}\right)=\left\{\frac{1}{m} \sum_{i=1}^{m} \operatorname{pr}\left(\mathbf{y} \mid \boldsymbol{\theta}^{(i)}\right)^{-1}\right\}^{-1}
$$

where $\theta^{(i)}$ denotes the $i$ th posterior draw of the parameter vector $\boldsymbol{\theta}$ of model $k$, and $m$ denotes the total number of posterior draws

\footnotetext{
${ }^{10}$ Equation 11, p. 779, Kass and Raftery (1995).
}

after the burn-in, equal to 5,000 in this calculation. The Bayes factor $B_{12}$ for comparing the modified with the regular Just-Pope model is obtained as

$$
B_{12}=\widehat{p r}\left(y \mid M_{1}\right) / \widehat{p r}\left(y \mid M_{2}\right) .
$$

We consider twice the natural logarithm of the Bayes factor, i.e., $2 \log _{e}\left(B_{12}\right)$, as the model selection criterion, which is on the same scale as the likelihood ratio test statistics (Kass and Raftery 1995). Table 2 calculates the statistic $2 \log _{e}\left(B_{12}\right)$ for the four datasets employed in this study. The results clearly reveal that the modified Just-Pope model is strongly favored relative to the regular Just-Pope model for datasets A, B, and C. For dataset D, our marginal likelihood calculations weakly support our modified Just-Pope model over the regular model.

\section{Quantile Regression Analysis of Skewness Shifts}

Estimation results of the quantile regression model are reported at nine quantiles ranging from 0.025 to 0.975 in the Supplemental Materials. All explanatory variables are centered at sample means. Based on the estimated coefficients, skewness shifts in equation (9) are calculated and their standard errors are computed from 1,000 bootstrap samples. Table 3 presents the means and standard errors of the estimated skewness shifts induced by one more unit of nitrogen for the middle $100(1-2 p) \%$ of the conditional crop yield distribution.

The results in table 3 indicate that higher levels of nitrogen application slightly decrease right-skewness for most of the selected measures in datasets A and B. Estimated $S K S^{(p)}$ values in dataset $A$ are all significantly different from zero at the $1 \%$ significance level. In 
Table 3. Skewness Shifts by one more unit of nitrogen for the Middle $100(1-2 p) \%$ of Conditional Yield Distribution Estimated by Quantile Regressions

\begin{tabular}{|c|c|c|c|c|}
\hline \multicolumn{5}{|c|}{ Dataset $A$} \\
\hline$p$ & 0.25 & 0.1 & 0.05 & 0.025 \\
\hline$S^{(p)}$ & 0.00 & -0.16 & -0.17 & -0.17 \\
\hline$S K S^{(p)}$ & $\begin{array}{c}-2.2^{* * *} \\
(0.7)\end{array}$ & $\begin{array}{c}-2.0^{* * *} \\
(0.5)\end{array}$ & $\begin{array}{c}-1.7^{* * *} \\
(0.4)\end{array}$ & $\begin{array}{c}-2.0^{* * *} \\
(0.5)\end{array}$ \\
\hline \multicolumn{5}{|c|}{ Dataset $B$} \\
\hline$p$ & 0.25 & 0.1 & 0.05 & 0.025 \\
\hline$S^{(p)}$ & -0.14 & -0.34 & -0.41 & -0.42 \\
\hline$S K S^{(p)}$ & $\begin{array}{c}0.2 \\
(0.8)\end{array}$ & $\begin{array}{c}-1.2^{* *} \\
(0.6)\end{array}$ & $\begin{array}{c}-1.7^{* * *} \\
(0.5)\end{array}$ & $\begin{array}{l}-2.1^{* * *} \\
(0.5)\end{array}$ \\
\hline \multicolumn{5}{|c|}{ Dataset $C$} \\
\hline$p$ & 0.25 & 0.1 & 0.05 & 0.025 \\
\hline$S^{(p)}$ & 0.19 & 0.34 & 0.33 & 0.36 \\
\hline$S K S^{(p)}$ & $\begin{array}{c}-0.1 \\
(2.0)\end{array}$ & $\begin{array}{c}-0.7 \\
(1.7)\end{array}$ & $\begin{array}{c}1.1 \\
(1.5)\end{array}$ & $\begin{array}{l}1.0 \\
(1.4)\end{array}$ \\
\hline \multicolumn{5}{|c|}{ Dataset D } \\
\hline$p$ & 0.25 & 0.1 & 0.05 & 0.025 \\
\hline$S^{(p)}$ & -0.07 & 0.12 & 0.37 & 0.40 \\
\hline$S K S^{(p)}$ & $\begin{array}{c}1.6 \\
(3.9)\end{array}$ & $\begin{array}{c}1.7 \\
(2.6)\end{array}$ & $\begin{array}{l}1.6 \\
(2.5)\end{array}$ & $\begin{array}{l}1.5 \\
(2.4)\end{array}$ \\
\hline
\end{tabular}

Note: Single $\left({ }^{*}\right)$, double $\left({ }^{* *}\right)$, and triple $\left({ }^{* * *}\right)$ asterisks denote significance at $0.10,0.05$, and 0.01 levels, respectively.

dataset B we observe significant negative skewness shifts measured at the $80 \%, 90 \%$, and $95 \%$ quantiles of the yield distribution. But the measure of $S K S^{(p)}$ is not significantly different from zero at the middle $50 \%$ of the distribution, which is associated with relatively higher values of yields. The percentage decrease in skewness induced by one more unit of nitrogen range from $0.17 \%$ to $0.22 \%$ in dataset $\mathrm{A}$ and from $-0.02 \%$ to $0.21 \%$ in dataset B. On average, the magnitude of impact appears to be smaller in dataset $\mathrm{B}$.

Although not significant in general, the impacts in dataset $\mathrm{C}$ are not consistent across different portions of the yield population, which may be because of the relatively smaller sample size. For the middle $50 \%$ and $90 \%$ of the yield population, the effect of high nitrogen application is negative, i.e., reducing right-skewness, but the effect changes to positive for the $95 \%$ and $97.5 \%$ quantiles. A positive and small effect of nitrogen on cottonyield skewness is found in dataset $\mathrm{D}$, indicating that a more right-skewed yield distribution is associated with higher levels of nitrogen.

\section{Rotation Effect on Yield Skewness}

One concern with the generalized JustPope model and the corresponding estimation method we propose in equation (2) is that the effect of fertilizer on skewness might be confounded by other factors. This may arise because in the model higher moments of crop yields are specified as only a function of fertilizer. For this reason, results may be inconsistent with skewness as measured in the quantile regression model, where the effect of fertilizer on skewness is investigated after controlling for other covariates. To account for potential effects of other controlled variables, especially any rotation effect in this case, we perform the additional robustness exercise of estimating a different set of skewness measures for each combination of nitrogen application level and rotation in order to separate the effect of rotation on yield skewness. The results are presented in table 4.

We focus on the rotation effect for two reasons: $(a)$ it is reasonable to expect a rotation effect on skewness, as nitrogen carryover from soybean can be viewed as a substitute for applied fertilizer (e.g., Vanotti and Bundy 1995); (b) our review of the literature provides us with little guidance on how other controlled variables, including dummy variables for time and location within narrow regions, will affect yield distribution other than shifting the mean; and $(c)$ with a limited number of observations in each dataset, it is difficult to estimate separate skewness measures for high-dimensional controlled variables, and we encounter the general curse-of-dimensionality problem. In addition, only datasets $\mathrm{A}$ and $\mathrm{B}$ are employed because dataset $\mathrm{C}$ is for continuous corn and dataset $\mathrm{D}$ provides no rotation information.

The results in table 4 indicate that after controlling for a rotation effect, the estimated skewness measures show a pattern similar to that of table 1. In general, for corn-after-corn and also for corn-after-soybeans corn yield skewness declines with an increase in low levels of nitrogen use. But in no case does significant change in skewness result at higher nitrogen levels. Further, for any given nitrogen rate, corn-after-soybean is strongly more negatively skewed than corn-after-corn. Table 5 summarizes the difference. In dataset $\mathrm{A}$ the absolute difference declines uniformly as nitrogen increases, consistent with the view that corn rotation after a legume acts as a substitute for more nitrogen. In dataset $\mathrm{B}$ the absolute 
Table 4. Bayesian Posterior Mean and Standard Deviation (in parentheses) for Derived Skewness after Accounting for Rotation Effect

\section{Dataset $A$}

Rotation: Corn-Corn

Nitrogen

0

Skewness

0.410

$(0.085)$

Rotation: Corn-Soybean

Nitrogen

Skewness

$$
\begin{gathered}
0 \\
-0.109 \\
(0.061)
\end{gathered}
$$

Dataset $B$

Rotation: Corn-Corn

$\begin{array}{lc}\text { Nitrogen } & 0 \\ \text { Skewness } & 0.209 \\ & (0.091)\end{array}$

Nitrogen

Skewness

$$
\begin{gathered}
-0.328 \\
(0.073)
\end{gathered}
$$

25
0.048
$(0.074)$

$$
\begin{gathered}
50 \\
-0.175 \\
(0.085)
\end{gathered}
$$$$
150
$$$$
-0.260
$$$$
\text { (0.065) }
$$

240

$-0.065$

(0.068)

(0.068)

240

$-0.360$

(0.064)

Rotation: Corn-Soybean

\begin{tabular}{lccccc} 
Nitrogen & 0 & 25 & 50 & 75 & 100 \\
Skewness & -0.445 & -0.452 & -0.495 & -0.539 & -0.538 \\
& $(0.062)$ & $(0.065)$ & $(0.072)$ & $(0.082)$ & $(0.077)$ \\
Nitrogen & 125 & 150 & 200 & 250 & 300 \\
Skewness & -0.486 & -0.522 & -0.462 & -0.470 & -0.438 \\
& $(0.079)$ & $(0.082)$ & $(0.083)$ & $(0.086)$ & $(0.077)$ \\
\hline
\end{tabular}

Table 5. Rotation Effect on Skewness at Different Nitrogen Levels; Skewness under CornSoybean Less under Corn-Corn

\section{Dataset $A$}

Nitrogen

Difference

0
-0.519

Dataset B

Nitrogen

Difference

Nitrogen

Difference

0
-0.654
125
-0.158

difference generally declines with an increase in nitrogen until the application rate is about $100 \mathrm{lb}$./acre, so there is evidence to suggest that rotation also determines yield skewness.

\section{Conclusion}

To test Day's (1965) observations that more nitrogen tends to make skewness less positive or more negative, but only up to a point, we develop two approaches to assessing the role of nitrogen in determining yield skewness. One is a generalization of the Just-Pope technology implemented with Bayesian methods, while the other is a quantile regression approach.

Experimental plot datasets allow us to address any concerns about temporal and spatial aggregation in yield modeling. For corn yields, estimation results from both methods provide strong evidence that negative skewness is associated with nonzero nitrogen rates. In addition, more negative skewness is associated with more nitrogen, and some evidence is provided that a ceiling nitrogen level exists 
above which skewness does not change. There is weaker evidence for positively skewed cotton yield, and we found no discernible skewness pattern as nitrogen levels change.

What practical relevance is there for our findings? There is strong empirical evidence in favor of a risk management preference for positive skewness (e.g., Harvey and Siddique 2000), while Chiu (2005) provides qualified theoretical support for this preference. So, all else equal, including other moments, risk preferences are unlikely to motivate a larger choice of nitrogen. In any case, commercial use of nitrogen on U.S. Corn Belt corn typically exceeds the threshold above which we find a minimal skewness response.

Another perspective is that skewness affects insurance indemnities and the extent of crop insurance subsidy costs. Other moments being equal, a more negatively skewed distribution suggests larger indemnity payouts, so it is no surprise that crop insurance effects have motivated many inquiries into yield skewness. In an environment of high commodity prices and high crop insurance subsidy rates (Shields 2010), a fundamental understanding of skewness determinants should prove useful. Ultimately we see the work's practical relevance as identifying stylized facts that need to be accounted for if a theory of how yield distributions are shaped is to square with the facts.

Finally there is the matter of whether experimental crop yield data reflect commercial yield data disaggregated to the farm level. Did growth in the use of nitrogen over in commercial crop agriculture during the 20th century actually affect skewness? In light of table 4 , might a shift from a corn-soybean rotation to a corn-corn-soybean rotation in response to demand for corn ethanol increase skewness? In real production systems, crop sharing, yield insurance, and other risk management arrangements are likely to have an effect. Also, input use can sometimes be adapted intra-season in response to weather, price, and other signals. The literature to date provides us with little sense of whether these realities could create a significant divergence between what applies for yield trial data and for market outcomes.

In conclusion, we think that development of a fuller theory on how input constraints affect yield skewness will have to await empirical regularities emerging from studies on diverse crops grown in different production environments. Although perhaps most convenient to work with, nitrogen and rotation are not the only management practices that can be varied in a controlled manner. For example, controlled experimental data on conservation tillage practices are available (e.g., DeVuyst and Halvorson 2004). To the extent that conservation tillage promotes soil water storage, one might expect an effect on yield skewness in water-constrained cropping areas. Data on irrigation effects are also available (e.g., Eck 1984; Kim et al. 2008) where one would expect that an increase in availability of irrigation water will act to make yield skewness less positive or more negative.

\section{References}

Antle, J. M. 1983. Testing the Stochastic Structure of Production: A Flexible MomentBased Approach. Journal of Business \& Economic Statistics 1(3): 192-201.

Antle, J. M. 2010. Asymmetry, Partial Moments and Production Risk. American Journal of Agricultural Economics 92(5): 1294-1309.

Babcock, B. A., and D. A. Hennessy. 1996. Input Demand under Yield and Revenue Insurance. American Journal of Agricultural Economics 78(2): 416-427.

Chiu, W. H. 2005. Skewness Preference, Risk Aversion, and the Precedence Relations on Stochastic Changes. Management Science 51(12): 1816-1828.

Day, R. H. 1965. Probability Distributions of Field Crop Yields. Journal of Farm Economics 47(3): 713-741.

DeVuyst, E. A., and A. D. Halvorson. 2004. Economics of Annual Cropping versus Crop-Fallow in the Northern Great Plains as Influenced by Tillage and Nitrogen. Agronomy Journal 96(1): 148-153.

Di Falco, S., and J. P. Chavas. 2009. On Crop Biodiversity, Risk Exposure, and Food Security in the Highlands of Ethiopia. American Journal of Agricultural Economics 91(3): 599-611.

Eck, H. V. 1984. Irrigated Corn Yield Response to Nitrogen and Water. Agronomy Journal 76(3): 421-428.

Gardner, B. L. 2002. American Agriculture in the Twentieth Century: How it Flourished and What it Cost. Cambridge MA:Harvard University Press.

Gelman, A., J. B. Carlin, H. S. Stern, and D. B. Rubin. 2004. Bayesian Data Analysis, 2nd ed. Chapman \& Hall/CRC.

Goodwin, B. K., and A. P. Ker. 2002. Modeling Price and Yield Risk. In A Comprehensive 
Assessment of the Role of Risk in U.S. Agriculture, ed. R. E. Just and R. D. Pope, ch. 14. Norwell, MA: Kluwer Academic Publisher.

Greene, W. H. 2003. Econometric Analysis. Upper Saddle River, NJ: Prentice Hall.

Hao, L., and D. Q. Naiman. 2007. Quantile Regression. Thousand Oaks, CA: Sage Publications.

Harvey, C. R., and A. Siddique. 2000. Conditional Skewness in Asset Pricing Tests. Journal of Finance 55(3): 1263-1295.

Hennessy, D. A. 2006. On Monoculture and the Structure of Crop Rotations. American Journal of Agricultural Economics, 88(4): 900-914.

- 2009. Crop Yield Skewness under Law of the Minimum Technology. American Journal of Agricultural Economics 91(1): 197-208.

- 2010. Crop Yield Skewness and the Normal Distribution. Journal of Agricultural and Resource Economics 33(1): 34-52.

Just, R., and R. D. Pope. 1978. Stochastic Specification of Production Function and Economic Implication. Journal of Econometrics 7(1): 67-86.

Just, R. E., and R. D. Pope. 1979. Production Function Estimation and Related Risk Considerations. American Journal of Agricultural Economics 61(2): 277-284.

Just, R. E., and Q. Weninger. 1999. Are Crop Yields Normally Distributed? American Journal of Agricultural Economics 81(2): 287-304.

Kass, R. E., and A. E. Raftery. 1995. Bayes Factors. Journal of the American Statistical Association 90(430): 773-795.

Kim, K.-I., D. E. Clay, C.G. Carlson, S.A. Clay, and T. Trooien. 2008. Do Synergistic Relationships between Nitrogen and Water Influence the Ability of Corn to Use Nitrogen Derived from Fertilizer and Soil? Agronomy Journal 100(2): 551-556.

Koenker, R., and G. Bassett. 1978. Regression Quantiles. Econometrica 46(1): 33-50.

Koop, G., D. J. Poirier, and J. L. Tobias. 2007. Bayesian Econometric Methods. Cambridge, UK: University Press.

Koundouri, P., C. Nauges, and V. Tzouvelekas. 2006. Technology Adoption under Production Uncertainty: Theory and Application to Irrigation Technology. American Journal of Agricultural Economics 88(3): 657-670.
Koundouri, P., and N. Kourogenis. 2011. On the Distribution of Crop Yields: Does the Central Limit Theorem Apply? American Journal of Agricultural Economics 93(5): 1341-1357.

Mallarino, A. P., E. Ortiz-Torres, and K. Pecinovsky. 2004. Effects of Crop Rotation and Nitrogen Fertilization on Crop Production. Annual progress report, Northeast Research and Demonstration Farm, Iowa State University.

Mitchell, P. 2004. Nutrient Best Management Practice Insurance and Farmer Perceptions of Adoption Risk. Journal of Agricultural and Applied Economics 36(3): 657-673.

Nelson, C. H., and P. V. Preckel. 1989. The Conditional Beta Distribution as a Stochastic Production Function. American Journal of Agricultural Economics 71(2): 370-378.

O'Donnell, C. J., and T. J. Coelli. 2005. A Bayesian Approach to Imposing Curvature on Distance Functions. Journal of Econometrics 126(2): 496-523.

Ramirez, O. A., S. Misra, and J. Field. 2003. Crop Yield Distributions Revisited. American Journal of Agricultural Economics 85(1): 108-120.

Seo, S., P. Mitchell, and D. J. Leatham. 2005. Effects of Federal Risk Management Programs on Optimal Acreage Allocation and Nitrogen Use in a Texas CottonSorghum System. Journal of Agricultural and Applied Economics 37(3): 685-699.

Shields, D. A. 2010. Federal Crop Insurance: Background and Issues. Congressional Research Service, CRS Report for Congress, December 13, http://www.natio nalaglawcenter.org/assets/crs/R40532.pdf (accessed October 29, 2011).

Terrell, D. 1996. Incorporating Monotonicity and Concavity Conditions in Flexible Functional Forms. Journal of Applied Econometrics 11(2): 179-194.

Vanotti, M. B., and L. G. Bundy. 1995. Soybean Effects on Soil Nitrogen Availability in Crop Rotations. Agronomy Journal 87(4): 676-680.

van Zwet, W. R. 1964. Convex Transformations of Random Variables. Amsterdam: Matematisch Centrum.

Yu, T., and B. A. Babcock. 2010. Are U.S. Corn and Soybeans Becoming More Drought Tolerant? American Journal of Agricultural Economics 92(5): 1310-1323. 
lowa State University does not discriminate on the basis of race, color, age, religion, national origin, sexual orientation, gender identity, genetic information, sex, marital status, disability, or status as a U.S. veteran. Inquiries can be directed to the Director of Equal Opportunity and Compliance, 3280 Beardshear Hall, (515) 294-7612. 